\title{
A quick and reliable waiting room checklist for symptoms of Disinhibited Social Engagement Disorder
}

\begin{abstract}
Background: Disinhibited Social Engagement Disorder (DSED) is a psychosocial disorder, associated with child maltreatment, characterised by indiscriminate friendliness towards strangers. Some behavioural overlap between DSED and Autism Spectrum Disorder (ASD) - a neurodevelopmental condition whose core symptoms include impaired communication - has been observed. Since DSED is associated with a maltreatment history and ASD is not, differential diagnosis is important.

Method: Norms and reference ranges are provided for a clinic waiting room scale (WRO) for observation of DSED symptoms based on two samples of children aged 5-12: 54 with DSED symptoms and 151 typically developing controls. We examined the WRO's ability to discriminate between children with DSED symptoms and with ASD through logistic regressions conducted on these samples, plus children with ASD $(n=16)$. Combining this analysis with qualitative observations of children with DSED symptoms $(n=5), \operatorname{ASD}(n=6)$ and controls $(n=7)$, we modified the measure to improve discrimination between these presentations.
\end{abstract}

Results: A WRO total score of <31 may indicate a multi-informant assessment for DSED. Children with ASD or DSED symptoms appeared more likely to approach strangers than controls; however, qualitative analysis of observations demonstrated that while children with DSED symptoms appeared to take control of the social aspects of the situation, children with ASD followed a nonsocial agenda, with the stranger appearing irrelevant. We modified the WRO to aid formulation around differential diagnosis.

Conclusions: The WRO is an efficient tool that can contribute to formulations around a child's difficulties with social relationships.

\section{Introduction}

Abused and neglected children are at high risk of psychiatric problems (Hughes et al., 2017). Disinhibited Social Engagement Disorder (DSED) is one of two disorders, described in DSM 5, that are specifically associated with maltreatment (American Psychiatric Association, 2013). DSED emerges early in life, in the context of maltreatment, and can persist into adolescence and even adulthood, especially if placement in nurturing foster or adoptive care occurs late, or if foster placements are unstable (Guyon-Harris et al., 2018). The core symptom of DSED is indiscriminate behaviour, including reduced reticence with strangers and a tendency to wander off without checking back with attachment figures (American Psychiatric Association, 2013). In middle childhood, DSED is associated with significant functional impairment and is frequently comorbid with other child psychiatric diagnoses; especially Attention Deficit Hyperactivity Disorder, Conduct Disorder and Anxiety Disorders (Kocovska et al., 2012). However, DSED is easily missed in clinical situations since its core symptom, indiscriminate behaviour, may not provoke concern and might even be interpreted as confident, friendly behaviour.

Several observational measures have been developed that can reliably detect DSED behaviours: three of these, the Attachment Formation Rating Scale (Corval et al., 2018), the Rating for Inhibited Attachment Behavior (Zeanah et al., 2005) and the Disinhibited Social Behavior Observational Measure (Bruce, Tarullo \& Gunnar, 2009), are based on videotaped laboratory observational procedures and a fourth, the Stranger at the Door procedure (Gleason et al., 2011), is based on observations of a home visit. None of these procedures could be incorporated into day to day clinical work in child and adolescent mental health. It was the need to find a clinically useful, yet reliable, 
observational measure for DSED that encouraged us, a decade ago, to develop the Waiting Room Observation (McLaughlin, Espie \& Minnis, 2010).

The clinic waiting room, an unfamiliar setting with parent(s) and stranger(s) present, is ideal for observing indiscriminate behaviour in school-age children. Shyness (i.e. wariness in situations that the child perceives to be socially novel or where social evaluation could occur) is typical in young children (Poole \& Shmidt, 2020). A clinic waiting room provides both social novelty and the potential for social evaluation. The Waiting Room Observation (WRO), is a quick and easy observational scale for DSED symptoms for use as part of a multi-informant assessment, along with information from parents and teachers (McLaughlin et al., 2010). It has contributed to clinical research diagnoses of DSED internationally (Lehmann et al., 2018), but limited research on its psychometric properties (McLaughlin et al., 2010) has restricted its clinical usefulness.

Of particular interest is the ability of the WRO to discriminate between DSED and Autism Spectrum Disorder (ASD): a neurodevelopmental condition involving impaired communication and interaction, fixed interests, and repetitive behaviour (Diagnostic Statistical Manual, $5^{\text {th }}$ Ed, 2013). Since both DSED and ASD involve difficulties with social relationships (Sadiq et al., 2012), some behavioural overlap across the two presentations can be observed. Yet, behavioural observation has been suggested to be the best means by which to discriminate between the two presentations (Davidson et al., 2015). The WRO may therefore be a helpful tool in aiding differential diagnosis, but it is not yet known whether the WRO is able to differentiate between ASD and DSED. Such differential diagnosis is important; since DSED is associated with maltreatment, a diagnosis can have significant child protection ramifications, i.e. may warrant investigation of concerns around maltreatment. Furthermore, preliminary evidence suggests that interventions to support children with DSED and ASD may differ (Becker-Weidman, 2006; Mukkades et al., 2004).

Here, population norms and reference ranges for the WRO are developed (Phase One). In Phase 2, the ability of the WRO to discriminate between DSED and ASD is examined and qualitative behavioural observations are used to identify modifications to be made to the WRO to improve its discriminatory ability.

\section{Methods}

\section{Phase One}

Population norms and reference ranges are developed for the WRO, so clinicians can easily recognise children with likely symptoms of DSED who require a full multi-informant assessment.

With ethical approval from the University of Glasgow and local Education Departments:

- existing data were accessed on 54 children aged 5-12 with DSED, diagnosed using standardised multi-informant measures (Kocovska et al., 2012) including the WRO.

- 151 Typically Developing (TD) comparison children from local primary schools were group matched on age and gender with the DSED group.

- WRO scores were compared in the DSED and TD groups to develop reference ranges. 


\section{Setting for observation}

Study assessments were conducted in one of two typical clinic waiting rooms with space for several families, following a protocol similar to the original study (McLaughlin et al., 2010). The child and caregiver enter the waiting room, in which the "stranger" is already present. There are toys available for the child to play with, and the caregiver is provided with questionnaires to complete. The "stranger" unobtrusively observes the child's behaviour, interacting with them only if the child initiates interaction. Upon completion of the questionnaires (typically around 10 minutes), the child and caregiver are debriefed. With two strangers available, observations can be made while the parent completes the questionnaires or, with only one stranger, immediately after the waiting room episode. The WRO can be administered with minimal training (e.g. five minutes of explanation) by a wide range of professionals including nurses, trainee psychiatrists and medical students.

The crucial aspects of the procedure are that:

- $\quad$ the child is in the waiting room for the first time

- the parent is distracted by e.g. a questionnaire

- the child's behaviour with the stranger and parent is observed for 5 to 10 minutes.

In the current study, $11 \%$ percent $(n=17)$ of WROs were simultaneously completed independently by two observers to measure inter-rater reliability.

\section{Measures}

The Waiting Room Observation (WRO) is a 17-item observer-rated observational scale for interactions between the child and stranger(s)/parent(s) on first meeting in an unfamiliar clinic waiting room (see Figure 1 for the full measure). In previous research with children aged 5-8 years it had good internal consistency (Cronbach alpha $=0.75$ ), good specificity and modest sensitivity in detecting children with DSED compared to typically developing control children (McLaughlin et al., 2010). Items are scored yes: no. For most items "yes" is atypical and is scored "0" with "no" typical and scored "1". Exceptions are items 6, 7, 11 and 17 which are reverse scored "yes: 1"; "no: 0". The scoring range of the WRO is 0 to 34 and a higher score indicates more typical waiting room behaviour.

Figure 1 about here.

The Strengths and Difficulties Questionnaire (SDQ) is a 25-item parent- and teacher-report screening instrument investigating common child mental health problems, well validated against other screening instruments and against psychiatric diagnosis (Goodman et al., 2004).

The Relationship Problems Questionnaire (RPQ) is a 10-item parent- and teacher-report screening instrument for Reactive Attachment Disorder (RAD) and DSED symptoms. In previous research it had good internal consistency (Cronbach's alpha $=0.85$ ) and factor analysis identified that six items describe inhibited RAD behaviours and four items describe DSED (Minnis et al., 2007). 


\section{Analysis}

Scores for the TD sample were computed on the RPQ, SDQ and WRO. Sensitivity analysis explored excluding children with a score above 7 on the RPQ because such a score indicates possible DSED. Inter-rater reliability was examined using the Bland-Altman Limits of Agreement Method (Giavarina, 2015) in which the mean difference between the ratings of two observers and the $95 \%$ confidence interval is calculated so that a judgement can be made as to whether any differences are unbiased and clinically acceptable.

Reference intervals were produced for the two samples. Data were normally distributed, so the reference interval was computed as $* \pm 1.96 \mathrm{~s}$, and confidence intervals for the upper and lower reference limits were computed using $\sqrt{3 \mathrm{~s}^{2} / \mathrm{n}}$ as an estimate of the standard error.

\section{Phase Two}

The ability of the WRO to discriminate between children with DSED and ASD was examined, and modifications to the WRO to improve its discriminatory ability were identified. With ethical approval from NHS West of Scotland Research Ethics Committee, we conducted a comparison of WRO data on:

- the TD sample described above ( $n=151$, plus 7 new cases; see below)

- the sample of children with DSED described above ( $n=54$, plus 5 new cases; see below)

- a sample of primary school aged children who already had a diagnosis of ASD recruited from NHS clinicians and third sector organisations ( $n=10$, plus 6 new cases; see below).

This analysis was supplemented by an observational study including the following primary school aged children:

- $\operatorname{TD}(n=7)$ recruited from local after school clubs

- with a diagnosis of ASD (made previously by clinicians using standard diagnostic procedures) ( $\mathrm{n}=$ 6) recruited from a third sector organisation providing support to family carers

- with DSED symptoms $(n=1)$ recruited from NHS clinicians, and video data of children $(n=4)$ who participated in a previous study (Minnis et al., 2016), whose scores on the Disturbances of Attachment Interview (Smyke \& Zeanah, 1999) (rated based on audio recordings of interviews by research nurses trained to good inter-rater reliability) suggested DSED, and whose caregivers provided consent for their data to be used.

The procedure for in-person observations followed that in Phase One. As well as the WRO, qualitative behavioural observations were taken by the stranger (researcher) and analysed to identify possible improvements to be made to the WRO to facilitate discrimination between DSED and ASD. A second stranger (researcher) was present for at least one observation out of every group of children (i.e. TD, DSED symptoms, or ASD), allowing one stranger to complete qualitative notes, and the other to complete the WRO, and facilitating discussion of the behaviours observed. A high degree of consensus was reached on these occasions. The video data (from 4 children with DSED symptoms) depicted the child and their caregiver taking part in a play/lunch session as part of another study (Minnis et al., 2016) in an unfamiliar clinic room. A researcher (stranger) entered the room at various points e.g. to provide the caregiver with expenses; the crucial aspects of the waiting room procedure were therefore similar in that the child was in an unfamiliar setting; their behaviour with the stranger/caregiver was observed, and the caregiver was at points distracted from the child. 
The recording was viewed, qualitative behavioural observations were taken and the WRO was completed by one researcher.

Analysis

WRO scores were analysed via multinomial logistic regression to identify the ability of each item to independently predict group membership of children with ASD and with DSED symptoms.

Qualitative behavioural observations were analysed using a grounded theory approach (Strauss \& Corbin, 1994), involving constant comparisons of cases within and between groups to generate themes that described the behaviours of each group of children. The same researcher completed the observation and qualitative analysis. Due to the recruitment procedures, the researcher who completed the observation and analysis was not blinded to the group membership (i.e. TD, DSED symptoms, or ASD) of participants during observations. This phenomenon may have influenced the interpretation of behaviours under observation. However, for at least one out of every group of participants, a second researcher (who was blinded to participant group) was present during the observation, and the two researchers compared findings. The researcher's role within and interpretation of observations was further reflected on within regular supervision sessions.

\section{Results}

\section{Phase One}

Reliability

Inter-rater reliability was excellent: the mean difference between the scores of two independent observers was small (.59 of a unit on the $0-34$ scale) and unbiased (limits of agreement -2.66 to 3.84).

\section{Characteristics of the samples}

Table 1 describes the characteristics of the sample. Higher mean SDQ and RPQ scores in the group of children with DSED suggest a greater degree of psychosocial difficulties generally in this sample compared to the TD group. One child in the TD group had an RPQ score of 16 and a WRO score of 21; during the observation of this child it was hypothesised that they may have ASD and as such would not be appropriate for the TD group, hence the case was removed from analyses. Four additional children in the TD group had RPQ scores of 7 or above (usually taken as indicating "screen positive" status for possible DSED - although this cut-off maximises sensitivity over specificity and gives a high false positive rate (Minnis et al., 2013)). Excluding those children from analyses made virtually no difference to the findings, therefore they were kept in the analysis. Group matching was successful for age but not gender (Table 1): the total WRO score was slightly but significantly higher in girls compared to boys (male 30.54 (3.09); female 31.89 (1.68), p<0.01) and the association between gender and participant group was significant $\left(\square^{2}(1)=6.18, p=0.017\right)$. In our TD sample, WRO scores were uncorrelated with area deprivation: a measure of average income, employment, education, health, access to services, crime and housing within small geographical areas of Scotland (Scottish 
Index of Multiple Deprivation https://www2.gov.scot/Resource/0050/00504809.pdf; Spearman's Rho .138; $p=.162$ ). We therefore controlled for gender in our regression analysis, but not for deprivation. Figure 2 displays the distribution of WRO total scores in the DSED and TD groups.

Table 1 about here.

Figure 2 about here.

A ROC curve (Figure 3 ) shows that the WRO is a "fair" test of DSED (area under the curve $=0.724$; 95\% Cl 0.629-0.819; $\mathrm{p}<0.001$ ) (Jayawant, 2010). A cut-off at a score of 31 or less gives a true positive rate of 0.622 and a false positive rate of 0.387 . In a binary logistic regression, $83.6 \%$ of children were correctly classified into the DSED or TD groups.

Figure 3 about here.

Table 2 details the six WRO items that best discriminate between TD children and those with DSED symptoms. We recommend a reference range for the total WRO score of 32-34 for typically developing children and that scores from 0-31 suggest the need for a multi-informant diagnostic assessment for DSED.

Table 2 about here.

\section{Phase Two}

Six WRO items significantly predicted group (DSED; ASD; TD) membership, with:
a) moves towards stranger,
b) does not respond reciprocally in conversation with carer,
c) adopts role of babyish child and
d) does not show preferential interest in carer's attention

predicting having ASD compared to TD group membership, and:
a) moves towards stranger,
b) does not display noticeable caution or shyness with stranger,
c) refuses or ignores request from carer,
d) adopts role of babyish child, and
e) does not show preferential interest in carer's attention

predicting having symptoms of DSED compared to TD group membership

The three items:
a) moves towards stranger,
b) adopts role of babyish child, and
c) does not show preferential interest in carer's attention

were therefore predictive of both ASD and symptoms of DSED. Does not respond reciprocally in 
conversation with carer was predictive of ASD but not symptoms of DSED, when compared to the TD group. Does not display noticeable caution or shyness with stranger and refuses or ignores request from carer was predictive of DSED symptoms but not ASD. Table 3 describes the six WRO items that best discriminated between the DSED, ASD and TD groups.

Table 3 about here.

Table 4 details the characteristics of the observational study sample. Higher mean SDQ and RPQ scores in the groups of children with ASD and with symptoms of DSED suggest a greater degree of psychosocial difficulties generally in these samples compared to the TD group. The qualitative analysis revealed important distinctions in the quality of the three behaviours that predicted both ASD and symptoms of DSED group membership. Table 5 outlines the themes identified in the qualitative analysis; some of which were independent of WRO items.

Table 4 about here.

Table 5 about here.

- Moves towards stranger. Children with DSED symptoms did so in the pursuit of an interaction with the stranger, e.g. to ask them a question or to engage in play with them. Children with ASD did so in pursuit of their own non-social agenda, e.g. to reach a toy, with a limited awareness of personal space as opposed to approaching the stranger to interact.

- Does not show preferential interest for carer's attention. Children with DSED symptoms showed an interest in interacting with the stranger. While some children with ASD showed a similar interest, this was exclusively for the purpose of talking about their special interest. Others with ASD showed no interest in interacting with anyone at all, and hence no preference for their carer's attention. Notably, does not respond reciprocally with carer predicted having ASD, which reinforces the lack of interaction generally in this group.

- Adopts role of babyish child. This was noted in both groups, was similar in quality and may be a genuine area of overlap among children with ASD/symptoms of DSED.

The key difference discriminating between ASD and symptoms of DSED appears to be the purpose of the interaction with strangers. In children with ASD, the stranger appears irrelevant, with children pursuing their own non-social agenda and interacting with the stranger (and sometimes the caregiver) only if it is helpful to that agenda. By contrast, children with DSED symptoms appear to cope with the stress of the unfamiliar situation, including the stranger's presence, by making social approaches to the stranger. Adding caveats outlining these differences to these items in the WRO may help to guide clinicians in scoring the WRO to discriminate between ASD and DSED symptoms (see Figure 4 for modified WRO including these caveats).

Figure 4 about here.

\section{Discussion}

In Phase One, we aimed to establish population norms and reference ranges for the WRO, to support its use by clinicians investigating DSED and to support multi-informant clinical and research diagnoses. To make a diagnosis of DSED, we would recommend its use alongside information from parents and teachers, as for any child psychiatric diagnosis. We found that the WRO is, in itself, a 
"fair" test for DSED i.e. it has reasonable discriminant validity in a clinical setting. We are not suggesting that it be used as a screening tool, but as a simple first step towards clinical diagnosis: our suggested cut-off point of 31 was chosen to prioritise sensitivity over specificity, since the WRO is likely to be the first assessment tool used when investigating DSED and information gathered later from parents and teachers is likely to rule out false positives. Tools such as the Reactive Attachment Disorder and Disinhibited Social Engagement Disorder Assessment (RADA) and teacher Relationship Problems Questionnaire may be useful as an adjunct to clinical assessment (Lehmann et al., 2018). A cut-off score of 31 or less on the WRO would suggest that a full multi-informant assessment for DSED is indicated.

A clinic waiting room is an ideal place to detect symptoms of DSED as it is an unfamiliar and therefore stressful environment for most children and presents an opportunity for children to interact with strangers, allowing core symptoms of DSED to be observed. This study, and the original WRO development study (McLaughlin et al., 2010), have demonstrated that children of primary school age sampled from the general population, regardless of social deprivation, exhibit marked shyness in an unfamiliar clinic waiting room. We found it striking to see how typically developing children "shut down" in this stressful setting, rarely making any approach towards strangers. Even conversation with their own caregiver in the waiting room is uncommon. In contrast, children with DSED symptoms usually continue to respond reciprocally with their caregiver despite being in this stressful new environment and make some approach, or at least look towards, the stranger. Our finding that a minority of children with DSED show some degree of shyness in the waiting room is perhaps unsurprising in such a stressful environment, and this perhaps analogous to the way some children with ADHD may show little hyperactivity on their first visit to the clinic. An important consideration is the familiarity of clinic waiting rooms to children who may have significant contact with services throughout their childhood, for example those in the care of Community Paediatric Services. The unfamiliar - and hence stressful - nature of the waiting room environment is considered important within this paradigm; its applicability to children already familiar with the setting may therefore be limited. However, the presence of unfamiliar strangers in a familiar clinic waiting room setting may trigger similar behaviours in children as those described here. This is an avenue for future research.

In Phase 2, we aimed to examine the ability of the WRO to discriminate between cases with DSED symptoms and with ASD, and to improve its ability to do so using qualitative behavioural observations. Our mixed methods approach demonstrates that, compared to typically developing children, both children with DSED symptoms and with ASD show less hesitancy in interacting with strangers in the waiting room paradigm. However, important distinctions in the nature of the interaction can be observed. Children with ASD appear to follow their own non-social agenda, e.g. playing with toys or talking about their special interest, with the stranger appearing irrelevant to this endeavour. In contrast, children with DSED symptoms appear to fail to reference their caregiver in the context of the unfamiliar situation, instead coping by taking control of the social interaction and making social approaches to the stranger. In contrast, typically developing children appear too inhibited by the stressful nature of the situation to interact with the stranger, staying close to their caregiver.

These findings correspond to theories on attachment in that as well as typically developing children, children with ASD display typical attachment behaviours (Teague et al., 2017), but they may display abnormal social interactions in that their shyness in an unfamiliar setting may be overridden by factors such as cues related to their special interest. In contrast, children with DSED symptoms do not reference their caregiver when in the waiting room, possibly failing to understand the hierarchical nature of attachment relationships within this paradigm, i.e. that the adults in the room (stranger and caregiver) typically control the social interaction. 
A significant number of children with a history of abuse and neglect may also meet criteria for neurodevelopmental vulnerabilities such as ASD (e.g. Kocovska et al., 2012; Mayes et al., 2016). As such, it is possible that a proportion of the sample with symptoms of DSED analysed here would also meet criteria for ASD, or indeed for other diagnoses. However, the purpose of the WRO is to aid clinicians' thinking in cases that are diagnostically difficult, including when symptoms of different diagnoses co-occur.

Our study is limited by the use of video data alongside in-person observations for the observational data. However, the key components of the waiting room procedure are evident in the protocol depicted by the video data. Additionally, the ASD sample, as well as the samples for the observational component of the study, were small. As such, further investigation is warranted with larger samples. Another important next step is to test the ability of the modified WRO to differentiate between ASD and symptoms of DSED.

\section{Conclusions}

Our research confirms that shyness is typical in primary school-aged children and that lack of shyness is a key symptom that might indicate a history of maltreatment. In order to aid differential diagnosis between ASD and DSED, attention must be paid to the function of the child's approach to strangers, i.e. whether to pursue a non-social agenda, or to control the social aspects of the situation. The modified WRO attempts to capture these nuances, providing prompts to aid clinicians' thinking around cases that are diagnostically difficult. An unfamiliar clinic waiting room is a perfect place to detect symptoms of DSED because it is a stressful environment that should induce shyness in a typically developing child. The WRO can be conducted and reliably scored in around 5-10 minutes by any member of staff who has had minimal training. This study has, therefore, provided clinicians with a highly efficient tool to aid diagnosis of DSED as part of a multi-informant diagnostic assessment.

\section{Acknowledgements}

We thank our participants, Glasgow Children's Hospital Charity for funding the study and Caitlin Bell, Nasreen Moya, Rebecca Nelson, Rachel Whyte and Calum Calderwood for help with data collection. The author(s) have declared that they have no competing or potential conflicts of interest. 
Reference List

American Psychiatric Association. (2013) Diagnostic and Statistical Manual of Mental Disorders DSM5. (Fifth Edition) Washington DC, London, England: American Psychiatric Publishing

Becker-Weidman, A. (2006) Dyadic developmental psychotherapy: a multi-year follow-up. New Developments in Child Abuse Research: 43, 60

Bruce, J., Tarullo, A.R., Gunnar, M.R. (2009) Disinhibited social behaviour among internationally adopted children. Development and Psychopathology: 21 157-171

Corval, R., Belsky, J., Baptista, J., Mesquita, A., Soares, I. (2018) Development and validation of an observational measure of symptoms of reactive attachment disorder. Attachment and Human Development: 21 111-131

Davidson, C., O'Hare, A., Mactaggart, F., Green, J., Young, D., Gillberg, C., Minnis, H. (2015) Social relationship difficulties in autism and reactive attachment disorder: Improving diagnostic validity through structured assessment. Research in Developmental Disabilities: 40 63-72

DSM-5, 2013. Diagnostic and statistical manual of mental disorders (5th ed). Washington, DC: American Psychiatric Association, 2013

Giavarina, D. (2015) Understanding bland altman analysis. Biochemia medica, 25 (2), 141-51

Gleason, M.M., Fox, N.A., Drury, S.S., Smyke, A.T., Egger, H.L., Nelson, C.A., Zeanah, C.H. (2011) Validity of evidence-derived criteria for reactive attachment disorder: Indiscriminately social/disinhibited and emotionally withdrawn/inhibited types. Journal of the American Academy of Child and Adolescent Psychiatry: 50 216-231

Goodman, R., Ford, T., Corbin, T., Meltzer, H. (2004) Using the Strengths and Difficulties Questionnaire (SDQ) multi-informant algorithm to screen looked-after children for psychiatric disorders. European Child \& Adolescent Psychiatry: 13(2 supplement):ii25-ii31

Guyon-Harris, K.L., Humphreys, K.L., Fox, N.A., Nelson, C.A., Zeanah, C.H. (2018) Course of disinhibited social engagement disorder from early childhood to early adolescence. Journal of the American Academy of Child \& Adolescent Psychiatry: 57(5), 329-335

Hughes, K., Bellis, M.A., Hardcastle, K.A., Sethi, D., Butchart, A., Mikton, C., et al. (2017) The effect of multiple adverse childhood experiences on health: a systematic review and meta-analysis. The Lancet Public Health: 2 (8), 356-366

Mandrekar, J.N. (2010) Receiver operating characteristic curve in diagnostic test assessment. Journal of Thoracic Oncology: 5 (9) 1315

Kocovska, E., Puckering, C., Follan, M., Smillie, M., Gorski, C., Barnes, J., et al. (2012) Neurodevelopmental problems in maltreated children referred with indiscriminate friendliness. Research in Developmental Disabilities: 33, 1560-1565

Lehmann, S., Monette, S., Egger, H., Breivik, K., Young, D., Davidson, C., Minnis, H. (2018) Development and examination of the Reactive Attachment Disorder and Disinhibited Social Engagement Disorder Assessment Interview. Assessment: 27 749-765 
Mayes, S.D., Calhoun, S.L., Waschbusch, D.A., Baweja, R. (2016) Autism and reactive attachment/disinhibited social engagement disorders: Co-occurrence and differentiation. Clinical Child Psychology and Psychiatry: 22 620-631

McLaughlin, A., Espie, C., Minnis, H. (2010) Development of a Brief Waiting Room Observation for Behaviours Typical of Reactive Attachment Disorder. Child and Adolescent Mental Health: 15 (2), 7379

Minnis, H., Boyd, K., Fitzpatrick, B., Forde, M., Gilberg, C., Henderson, M., McMahon, L., McIntosh, E., McConnachie, A., Messow, M., Millar, S., Moore, L., Ougrin, D., Watson, N., Wilson, P. (2016) Protocol 15PRT/6090: The Best Services Trial (BeST?): effectiveness and cost-effectiveness of the New Orleans Intervention Model for Infant Mental Health - NCT02653716 The Lancet

Minnis, H., MacMillan, S., Pritchett, R., Young, D., Wallace, B., Butcher, J., Sim, F., Baynham, K., Davidson, C., Gillberg, C. (2013) Prevalence of reactive attachment disorder in a deprived population. The British Journal of Psychiatry: 202 342-346

Minnis, H., Reekie, J., Young, D., O'Connor, T., Ronald, A., Gray, A., et al. (2007) Genetic, environmental and gender influences on attachment disorder behaviours. British Journal of Psychiatry: 190 (490), 495

Mukkades, N.M., Kaynak, F.N., Kinali, G., Besikci, H., Issever, H. (2004) Psychoeducational treatment of children with autism and reactive attachment disorder. Autism: 8 101-109

Poole, K.L., Shmidt, L.A. (2020) Positive shyness in the brain: Frontal electroencephalogram alpha asymmetry and delta-beta correlation in children. Child Development: 91 (5) e1030-31045

Sadiq, F., Slator, L., Skuse, D., Law, J., Gillberg, C., Minnis, H. (2012) Social use of language in children with reactive attachment disorder and autism spectrum disorders. European Child and Adolescent Psychiatry: 41 972-982

Smyke, A.T., Zeanah, C.H. (1999) Disturbances of Attachment Interview. Unpublished manuscript

Strauss, A., Corbin, J. (1994) Grounded Theory Methodology (I) Handbook of Qualitative Research: 17 273-285

Teague, S.J., Gray, K.M., Tonge, B.J., Newman, L.K. (2017) Attachment in children with autism spectrum disorder: A systematic review. Research in Autism Spectrum Disorders, 35 35-50

Zeanah, C.H., Smyke, A.T., Koga, S.F., Carlson, E.A. \& the BEIP Group (2005) Attachment in institutionalized and community children in Romania. Child Development: 76101501528 


\section{Waiting Room Observation}

Participant No:

Additional observations/comments

\section{Child stranger interaction}

1. Does the child look at stranger(s) as if to invite conversation?

(The child does not have to smile but, eye contact must be of a quality that would

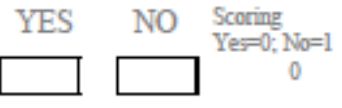
invite the stranger to commumicate in a "normal" social setting.)

2. Does the child interrupt conversation between the stranger(s) and his/her carer?

3. Does the child initiate conversation with the stranger(s) as if previously familiar?

4. Does the child move towards and approach the stranger(s)?

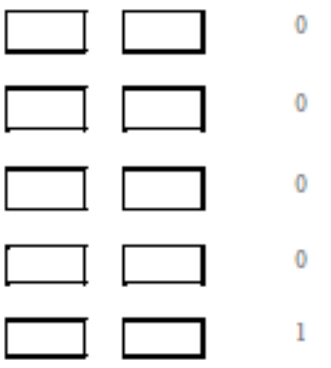

6. Does the child display noticeable caution or shyness with the stranger(s)?

\section{Child-carer interaction}

7. Is there a warmth to the carer-child relationship eg handholding, leaning in or cosy whispered chat, without parental irritation? (Does not have to include physical contact.)

8. Does the child make spontaneous comment to his/her carer in the presence of the introduced stranger (researcher one)?

9. Does the child refuse or ignore a request from his/her carer?

10. Does the child exhibit hyper compliance (defined as complying immediately, without question; seemingly eager to please) to a request from his/her carer?

11. Does the child respond reciprocally in conversation with his/her carer?

\section{General behaviours}

12. Does the child display rapid shifts in emotional expression (defined as sudden shifts to the extremes of emotion)?

13. Does the child appear to adopt the role of playing a younger, "babyish" child either in voice or behaviour?

14. Does the child appear superficially charming i.e. affection appears insincere or over the top (e.g. gives a false smile)?

15. Does the child try to exert control over their environment (e.g. tries to be the boss)?

16. Does the child display a seemingly insatiable desire for attention?
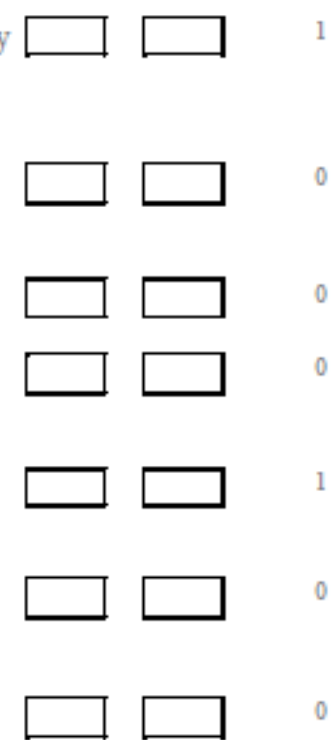

17. Does the child show a preferential interest in his/her carer's attention over that of the stranger's? 
Table 1: Comparison of DSED and Typically Developing groups

\begin{tabular}{|c|c|c|c|}
\hline & DSED (54) & $\begin{array}{c}\text { Typically } \\
\text { Developing (150) }\end{array}$ & \\
\hline & \multicolumn{3}{|c|}{ Mean (SD) and t test except where stated } \\
\hline Gender (\% Female) & $36 \%$ & $57 \%$ & $\mathrm{p}=.012\left(\mathrm{Chi}^{2}\right)$ \\
\hline Age & 7.84 & 7.94 & $p=.782$ \\
\hline Waiting Room Observation score & $\begin{array}{l}29.13 \text { (3.81) } \\
\text { Median } 30 \\
\text { Range 18-34 } \\
\text { IQR 27-32 }\end{array}$ & $\begin{array}{l}31.80 \text { (1.78) } \\
\text { Median } 32 \\
\text { Range 28-34 } \\
\text { IQR 31-33 }\end{array}$ & $p<.0001$ \\
\hline $\begin{array}{r}\text { Relationship Problems Questionnaire } \\
\text { Score }\end{array}$ & $\begin{array}{l}\text { Median } 11 \\
\text { Range 0-25 } \\
\text { IQR 4.5-17 }\end{array}$ & $\begin{array}{l}\text { Median } 0 \\
\text { Range 0-13 } \\
\text { IQR 0-2 }\end{array}$ & $\begin{array}{l}\mathrm{p}<.0001 \\
\text { (Mann Whitney) }\end{array}$ \\
\hline Strengths and Difficulties Questionnaire & $20.44(7.63)$ & $6.69(4.8)$ & $\mathrm{p}<.0001$ \\
\hline
\end{tabular}

Figure 2: Boxplot comparing Waiting Room Observation Scale (Obs schedule) total score in Typically Developing and DSED groups

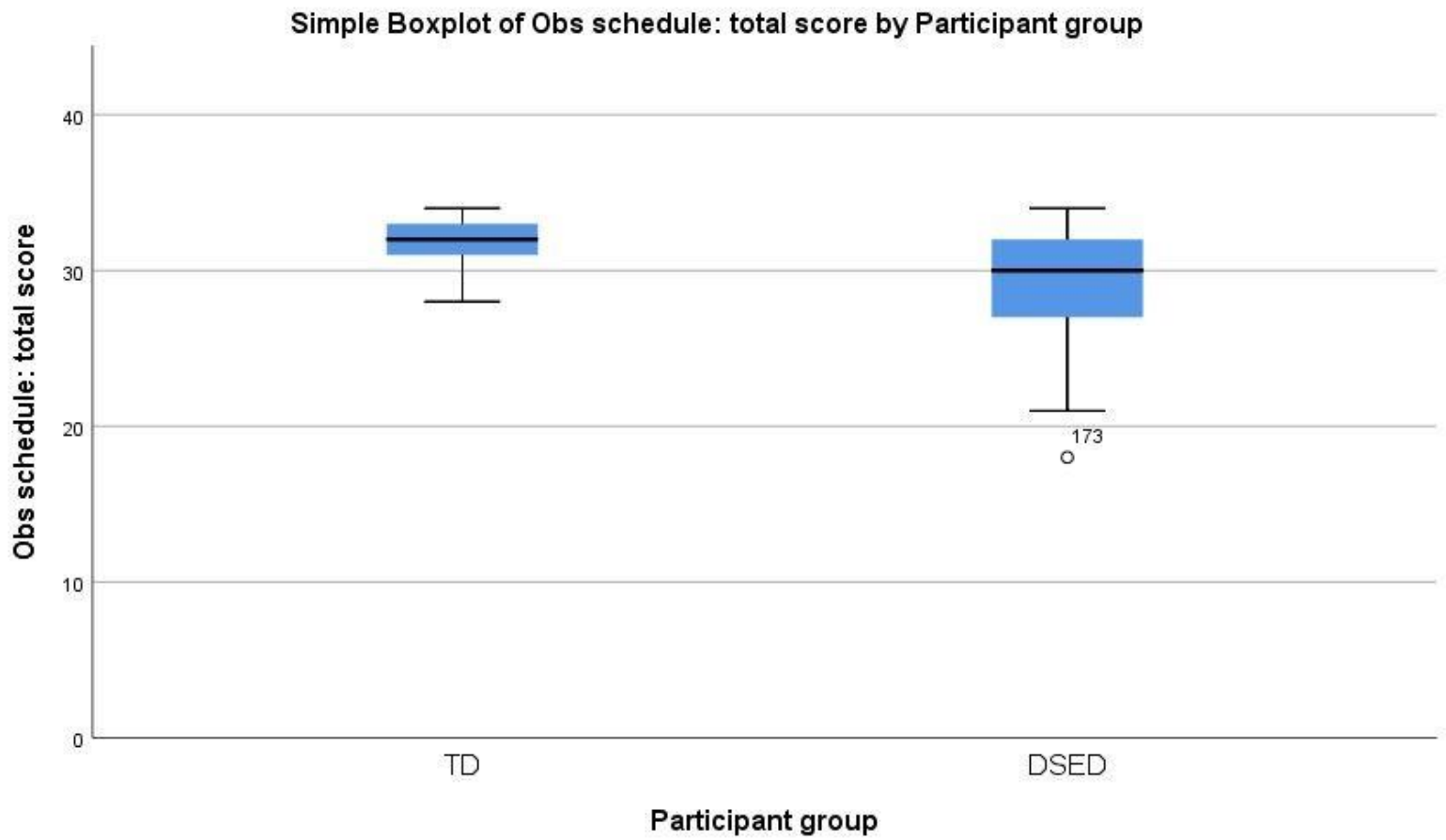


Figure 3: ROC Curve

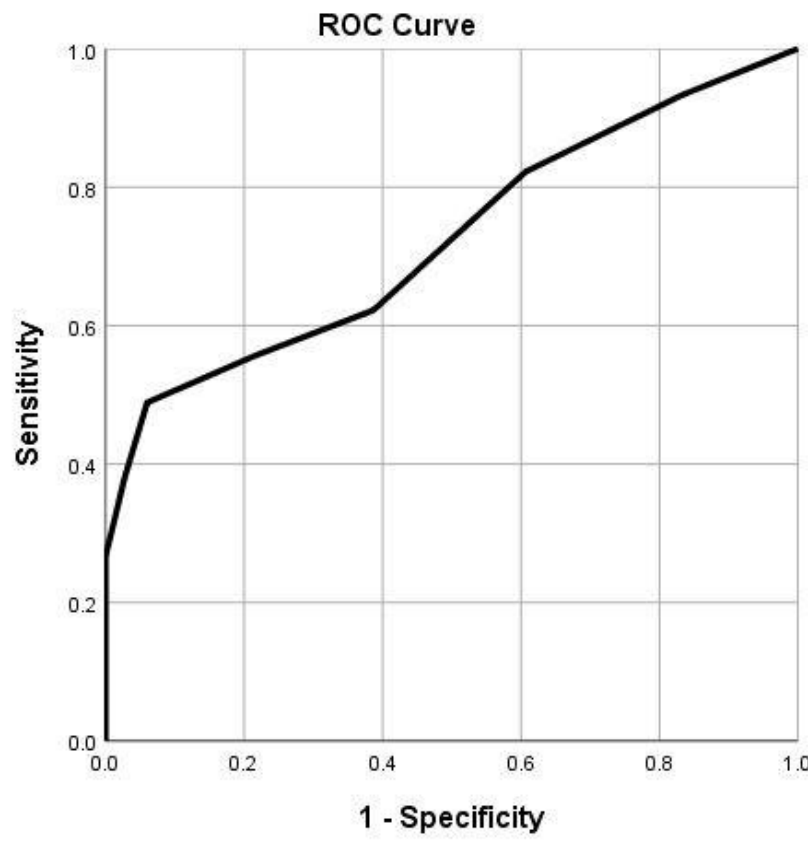

Diagonal segments are produced by ties.

Table 2: The 6 items best discriminating between DSED and Typically Developing Groups

\begin{tabular}{|l|l|l|l|}
\cline { 2 - 4 } \multicolumn{1}{c|}{} & \multicolumn{2}{c|}{ Percentage endorsing item } & Difference \\
\hline Displays insatiable desire for attention & 37.8 & 2.0 & 35.8 \\
\hline Refuses or ignores request from carer & 40 & 4.6 & 35.4 \\
\hline $\begin{array}{l}\text { Displays noticeable caution or shyness with } \\
\text { stranger(s) N.B. low shyness indicates DSED }\end{array}$ & 26.7 & 57.3 & 30.6 \\
\hline $\begin{array}{l}\text { Does not show preferential interest for carer's } \\
\text { attention }\end{array}$ & 80.0 & 54.3 & 25.7 \\
\hline Moves towards stranger(s) & 28.8 & 2.6 & 26.2 \\
\hline Interrupts conversation between carer and stranger & 26.7 & 18.2 & 8.5 \\
\hline
\end{tabular}


Table 3: The 6 items best discriminating between DSED/ASD and Typically Developing Groups

\begin{tabular}{|l|l|l|l|}
\cline { 2 - 4 } \multicolumn{1}{l|}{} & \multicolumn{3}{c|}{ Percentage endorsing item } \\
\cline { 2 - 4 } & \multicolumn{1}{c|}{ DSED (59) } & \multicolumn{1}{c|}{ ASD (16) } & $\begin{array}{c}\text { Typically } \\
\text { developing } \\
\text { (158) }\end{array}$ \\
\hline Moves towards stranger(s) & 35.6 & 25.0 & 3.8 \\
\hline Responds reciprocally in conversation with carer & 91.5 & 50.0 & 87.3 \\
\hline $\begin{array}{l}\text { Displays noticeable caution or shyness with } \\
\text { stranger(s) N.B. low shyness indicates DSED }\end{array}$ & 25.4 & 62.5 & 57.6 \\
\hline $\begin{array}{l}\text { Shows preferential interest for carer's attention } \\
\text { N.B. lack of preference for carer's attention } \\
\text { indicates DSED }\end{array}$ & 74.6 & 68.8 & 56.3 \\
\hline \begin{tabular}{l} 
Adopts role of babyish child \\
\hline Refuses or ignores request from carer
\end{tabular} & 25.4 & 25.0 & 0.6 \\
\hline
\end{tabular}

Table 4: Observational study sample characteristics

\begin{tabular}{|c|c|c|c|}
\hline & DSED (5) & $\begin{array}{c}\text { Typically } \\
\text { Developing (7) }\end{array}$ & ASD (6) \\
\hline & \multicolumn{3}{|c|}{ Mean (SD) } \\
\hline Gender (\% Female) & 60.0 & 85.7 & 0.0 \\
\hline Age & 5.6 & 7.6 & 9.5 \\
\hline Waiting Room Observation score & $25.8(2.6)$ & $32.7(1.4)$ & $28.7(3.3)$ \\
\hline $\begin{array}{r}\text { Relationship Problems Questionnaire } \\
(\text { RPQ)/Disturbances of Attachment } \\
\text { Interview (DAI) Score }\end{array}$ & $\begin{array}{l}24 \text { (RPQ) } \\
6.5(1.0) \text { (DAI) }\end{array}$ & $0.4(0.8)$ & $13.3(6.7)$ \\
\hline Strengths and Difficulties Questionnaire & $21.0(5.2)$ & $3.1(2.4)$ & $24.2(6.0)$ \\
\hline
\end{tabular}


Table 5: Themes identified from qualitative analysis of behavioural observations

\begin{tabular}{|c|c|c|}
\hline Theme & Group & Description \\
\hline \multirow[t]{3}{*}{ Child-caregiver interaction } & TD & $\begin{array}{l}\text { Remained close to caregiver, often leaning into them while } \\
\text { completing forms. Often whispered and giggled together. }\end{array}$ \\
\hline & ASD & $\begin{array}{l}\text { Mostly sat by themselves and only came close to caregiver when } \\
\text { it was functional to do so i.e. to complete a form. } \\
\text { Laughed and smiled together. }\end{array}$ \\
\hline & DSED & $\begin{array}{l}\text { Disorganised i.e. seeking proximity and then pushing caregiver } \\
\text { away. Laughing and reciprocal interaction around } \\
\text { toys but children also controlled direction of play or disagreed } \\
\text { with caregivers. }\end{array}$ \\
\hline \multirow{4}{*}{ exploration of environment } & TD & $\begin{array}{l}\text { Some children approached toys straight away whereas some only } \\
\text { did so when prompted by caregiver. Some did not move from } \\
\text { beside the caregiver. Most moved around the room more over } \\
\text { time. }\end{array}$ \\
\hline & & \\
\hline & ASD & $\begin{array}{l}\text { Some did not move around room at all. Those that did approach } \\
\text { the toys and move around did so straight away with no } \\
\text { prompting from caregiver. }\end{array}$ \\
\hline & DSED & $\begin{array}{l}\text { Most moved around room with no prompting from } \\
\text { caregiver. Some were asked by caregivers to return to seat } \\
\text { due to interaction with items in room other than toys. }\end{array}$ \\
\hline \multirow[t]{3}{*}{ Child-stranger(s) interaction } & TD & $\begin{array}{l}\text { One sibling group made comments to stranger at beginning of } \\
\text { interaction. Majority did not attempt to make eye contact but } \\
\text { smiled and looked away if eye contact was made; invited more } \\
\text { interaction over time e.g. making eye contact. }\end{array}$ \\
\hline & ASD & $\begin{array}{l}\text { Some made no attempt to interact with stranger(s) but came } \\
\text { physically close to stranger(s) e.g. to retrieve a toy. Two invited } \\
\text { interaction with the stranger straight away e.g. talking about } \\
\text { special interests or family. }\end{array}$ \\
\hline & DSED & $\begin{array}{l}\text { Majority interacted with stranger(s) immediately, e.g. } \\
\text { asking questions about them or approaching them to play. Some } \\
\text { sought out stranger(s) when not present in the room e.g. asking } \\
\text { where they were or going to find them. }\end{array}$ \\
\hline $\begin{array}{l}\text { Child's spontaneous } \\
\text { comments/interruptions in } \\
\text { stranger(s)' presence }\end{array}$ & TD & $\begin{array}{l}\text { Minority made spontaneous comments in stranger(s)' presence } \\
\text { but tended to whisper if they did so. Some provided commentary } \\
\text { on their activity/play, often if interacting with a sibling. }\end{array}$ \\
\hline
\end{tabular}




\begin{tabular}{|l|l|l|}
\hline & ASD & $\begin{array}{l}\text { Some made no comments at all. Some made frequent } \\
\text { spontaneous comments related to procedures of the study or } \\
\text { later in the day. Some provided commentary on their } \\
\text { activity/play. Often shouted or played loudly with toys (e.g. } \\
\text { slamming toys). Some interrupted caregiver while distracted. }\end{array}$ \\
\hline DSED & $\begin{array}{l}\text { All made spontaneous comments in presence of stranger(s). } \\
\text { Often shouted or played loudly (e.g. slamming toys). Some } \\
\text { interrupted caregiver while distracted. }\end{array}$ \\
\hline
\end{tabular}


Figure 4: Modified WRO

Participant No:

\section{Waiting Room Observation}

Additional observations/comments

Child stranger interaction

1. Does the child look at stranger(s) as if to invite conversation?

(The child does not have to smile but, eye contact must be of a quality that would invite the stranger to communicate in a "normal" social setting.)

2. Does the child interrupt conversation between the stranger(s) and his /her carer?

3. Does the child initiate conversation with the stranger(s) as if previously familiar? (For a score of YES, the interaction must feel reciprocal and the child must show an intereat in the attention of the stranger(s) as opposed to e.g. giving a monologue about a special interest.)

4. Does the child move towards and approach the stranger(5)?

(For a score of YES, the child's approach must be accompanied by social eye contact interaction with the stranger(s) as opposed to e.g. reaching for a toy.)

5. Does the child make physical contact with the stranger(s)?

6. Does the child display noticeable caution or shyness with the stranger(5)?

\section{Child-carer interaction}

7. Is there a warmth to the carer-child relationship eg handholding, leaning in or cosy whispered chat, without parental irritation? (Does not have to include physical contact.)

8. Does the child make spontaneous comment to his/her carer in the presence of the introduced stranger (researcher one)?

9. Does the child refuse or ignore a request from his/her carer?

10 Does the child exhibit hyper compliance (defined as complying immediately, without question; geemingly eager to please) to a request from his/her carer?

Does the child respond reciprocally in conversation with his her carer?

General behavioural characteristics

12 Does the child display rapid shifts in emotional expression (defined as sudden shifts to the extremes of emotion)?

13 Does the child appear to adopt the role of playing a younger, "babyish" child either in woice or behaviour?

14 Does the child appear superficially charming i.e. affection appears insincere or over the top (e.g. gives a false gmile)?

15 Does the child try to exert control over their environment (e.g. tries to be the boss)?

16 Does the child display a seemingly insatiable deaire for attention?

17 Does the child show a preferential interest of his/her carer's attention over that of the stranger's? (For a score of NO, child must show preferential interest in the stranger's attention as opposed to that of the carer as opposed to no interest in either the stranger or the carer's attention.) 